\title{
Development of A Generic UPLC/MS Workflow for the Authentication of Hoodia Gardonii Based on Comprehensive Chemical Fingerprinting
}

\author{
Bharathi Avula ${ }^{1}$, Yan-Hong Wang ${ }^{1}$, Kate $\mathrm{Yu}^{2}$, Dominic Moore ${ }^{2}$, Warren Potts ${ }^{2}$ and Ikhlas A Khan ${ }^{1,3}$

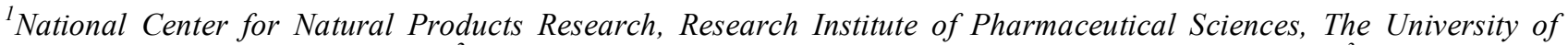 \\ Mississippi, MS 38677, USA; ${ }^{2}$ Waters Corporation, 34 Maple Street, Milford, MA, USA; ${ }^{3}$ Department of \\ Pharmacognosy, School of Pharmacy, The University of Mississippi, MS 38677, USA
}

Introduction: In recent years, Hoodia plant and the preparations of Hoodia have become increasingly popular. These dietary supplements are promoted for weight loss. The limited availability of this plant material and its increasing popularity leads to the possibility of adulterations by other species or even genera. Previous results of screenings give rise to serious concerns about the safety of commercial products claimed to be Hoodia, as a considerable amount seems to lack Hoodia. There is a strong need to develop an effective analytical protocol that can be routinely used to authenticate either plant extracts or commercial products that claimed to contain Hoodia gordonii.

Methods: Fourteen samples were used for this analysis. Of these fourteen samples, one known to be authentic Hoodia gordonii from South Africa, three Hoodia species from Missouri Botanical Garden, USA, three Hoodia gordonii samples from commercial source, five were commercially obtained dietary supplements claimed to contain Hoodia gordonii, and two were Opuntia species (spp.), an adulterant which grows quickly with no appetite suppressing activity associated. Samples were analyzed by an ACQUITY UPLC/Synapt G2 MS system with an UPLC HSS T3 column $(100 \mathrm{~mm} \times 2.1 \mathrm{~mm})$. The flow rate was rate was $0.6 \mathrm{~mL} / \mathrm{min}$ with water with $0.05 \%$ formic acid (A) and acetonitrile (B) as mobile phase. ESI+ and ESI- experiments were performed with data acquired over a range of $\mathrm{m} / \mathrm{z} 100$ to 1700 .

Preliminary Data: The first step of this workflow was to analyze these samples using a typical plant metabolomics analysis protocol, i.e.: UPLC/oaTOF MS with data indepent acquisition (no less than 3 injections per sample) for the entire sample set. Multi-Variate Statistical Analysis data processing strategy was used so that as initial results, a group of chemical markers can be generated, these markers are characteristics of Hoodia gardonii and were identified based on comprehensive chemical fingerprinting. If samples were analyzed in both positive and negative ionization modes, then two lists of chemical markers can be obtained, one from positive ESI and other from negative ESI. Some markers will be on both lists which help the confirmations; some markers will be on only one mode as compounds ionization behaviors differ. Our preliminary results were obtained based on PCA analysis for the entire sample set, followed by OPLS-DA analysis of authentic Hoodia gordonii vs different samples. There are a group of markers consistently appeared to be the key markers for Hoodia gordonii regardless what was the other group the Hoodia gordonii was compared against. In our results, in ESI-, we identified 11 markers as Hoodia gordonii markers, and in ESI+, we identified 30 markers as Hoodia gardonii markers. The second step of this workflow was to create a Hoodia gordonii LC/MS authentication method using the identified marker information. This method contains the name of the marker compound along with its expected LC retention time, exact molecular mass information, and MS-MS fragment information (optional). In this project, two methods were created, one based on ESI+ results, and one based on ESI- results. Each method can be used to test unknown samples based on which ionization mode was used during testing. For unknown sample that claims to contain Hoodia gordonii as an ingredient, one UPLC/MS injection was made, an application manager from the mass spec vendor allows east application of the Hoodia authentication method, as a result, a report was automatically generated to indicate how many of the essential markers were present in the unknown sample at what concentration level so that the authentication of the unknown sample can be easily accomplished.

Novel aspect: A generic workflow based on comprehensive chemical fingerprinting using UPLC/TOF MS ${ }^{\mathrm{E}}$ with novel informatics solution for plant authentication (Hoodia gordonii) 